\title{
Surgical antibiotic prophylaxis use and infection prevalence in non-cosmetic breast surgery procedures at a tertiary hospital in Western Australia - a retrospective study
}

Ainslie Lavers ${ }^{1}$, Wai Siong Yip ${ }^{1}$, Bruce Sunderland ${ }^{1}$, Richard Parsons ${ }^{1}$, Sarah MacKenzie ${ }^{2}$, Jason Seet ${ }^{2}$, Petra Czarniak ${ }^{\text {Corresp. } 1}$

${ }^{1}$ School of Pharmacy and Biomedical Sciences, Curtin University, Perth, Western Australia, Australia

2 Pharmacy Department, Sir Charles Gairdner Hospital, Perth, Western Australia, Australia

Corresponding Author: Petra Czarniak

Email address: P.Czarniak@curtin.edu.au

Background. Surgical site infections (SSIs) are a common complication following breast surgery procedures, despite being considered a clean surgery. The prevalence of SSIs can be minimised with the appropriate use of antibiotic prophylaxis as outlined in the Australian Therapeutic Guidelines (eTG). The aims of this study were to evaluate adherence to the eTG for antibiotic prophylaxis in breast surgery procedures at a Western Australian teaching hospital following an update of the guidelines in 2014 and examine the impact of prophylactic antibiotics on SSI incidence and length of hospital stay. Method. A retrospective cross-sectional study which reviewed medical records from a random sample of 250 patients selected from 973 patients who underwent breast surgical procedures between February 2015 and March 2017. Results. Overall adherence to current eTG occurred in 49.2\% (123/250) of operations. Pre-operative and post-operative antibiotics were prescribed in $98.4 \%$ (246/250) and $11.2 \%$ (28/250) operations respectively. Adherence rates to three specific elements of the eTG (drug prescribed, drug dosage and timing of administration) were $91.6 \%$ (2296/250), 53.6\% (134/250) and 86.4\% (216/250) respectively. For the $14.4 \%$ (36/250) patients with relevant drug allergies, there was zero adherence to the eTG. Overall recorded SSI prevalence was low at 5.2\% (13/250). The mean length of stay in patients ( $2.3 \pm 1.7$ days) was not influenced by level of eTG adherence $(p=0.131)$ or SSIs $(p=0.306)$. Conclusion. These data demonstrate a significant improvement in overall adherence to the eTG from $13.3 \%$ to $54.9 \%$ ( $p=$ $<0.001)$. The level of detected SSIs in this study was low. Further improvement is necessary with respect to prescribing appropriate antibiotic dosages and for those with allergies. 
1 Surgical antibiotic prophylaxis use and infection prevalence in non-cosmetic breast

2 surgery procedures at a tertiary hospital in Western Australia - a retrospective study

4

5

6 Ainslie Lavers ${ }^{1}$, Wai Siong Yip ${ }^{1}$, Bruce Sunderland ${ }^{1}$, Richard Parsons ${ }^{1}$, Sarah MacKenzie ${ }^{2}$, 7 Jason Seet $^{2}$, Petra Czarniak ${ }^{1}$

8

$9 \quad{ }^{1}$ School of Pharmacy and Biomedical Sciences, Curtin University, Perth, Western Australia.

10 2Pharmacy Department, Sir Charles Gairdner Hospital, Perth, Western Australia.

15 Corresponding Author:

16 Dr Petra Czarniak

17 P.Czarniak@curtin.edu.au 
19

20

21

22

23

24

\section{Abstract}

Background. Surgical site infections (SSIs) are a common complication following breast surgery procedures, despite being considered a clean surgery. The prevalence of SSIs can be minimised with the appropriate use of antibiotic prophylaxis as outlined in the Australian Therapeutic Guidelines (eTG). The aims of this study were to evaluate adherence to the eTG for antibiotic prophylaxis in breast surgery procedures at a Western Australian teaching hospital following an update of the guidelines in 2014 and examine the impact of prophylactic antibiotics on SSI incidence and length of hospital stay. Method. A retrospective cross-sectional study which reviewed medical records from a random sample of 250 patients selected from 973 patients who underwent breast surgical procedures between February 2015 and March 2017. Results. Overall adherence to current eTG occurred in 49.2\% (123/250) of operations. Pre-operative and postoperative antibiotics were prescribed in 98.4\% (246/250) and 11.2\% (28/250) operations respectively. Adherence rates to three specific elements of the eTG (drug prescribed, drug dosage and timing of administration) were 91.6\% (2296/250), 53.6\% (134/250) and 86.4\% (216/250) respectively. For the $14.4 \%$ (36/250) patients with relevant drug allergies, there was zero adherence to the eTG. Overall recorded SSI prevalence was low at 5.2\% (13/250). The mean length of stay in patients ( $2.3 \pm 1.7$ days) was not influenced by level of eTG adherence $(p=0.131)$ or SSIs $(p=0.306)$. Conclusion. These data demonstrate a significant improvement in overall adherence to the eTG from $13.3 \%$ to $54.9 \%(p=<0.001)$. The level of detected SSIs in this study was low. Further improvement is necessary with respect to prescribing appropriate antibiotic dosages and for those with allergies. 


\section{Introduction}

Surgical site infections (SSIs) are a common complication following breast surgery procedures, despite being considered a 'clean surgery' [1-2]. SSIs are also the second most common adverse event in breast surgical patients with incidences of infection typically ranging from $0.8-26 \%$ [1$3]$.

The prevalence of SSIs can be minimized with the appropriate use of pre-operative antibiotic prophylaxis [4-5]. This has been demonstrated in patients undergoing breast surgery [6]. A recent study by Jaber et al., in 2014 evaluated the appropriateness of surgical antibiotic prophylaxis for breast surgery procedures and found a statistically significant relationship between pre-operative prophylactic antibiotic use and successful SSI prevention [6]. The burden of SSIs not only includes an impact on patient recovery, the associated cost of hospital readmission and subsequent adjuvant treatment, but also SSI-related patient morbidity and mortality. Hence the application and adherence to evidence-based guidelines should be considered to minimize rates of SSIs [1, 7-8].

In Australia, prescribers are guided by the Australian Antibiotic Therapeutic Guidelines (eTG) which aim to promote the quality use of medicines by publishing evidence based standard protocols and up-to-date therapeutic information. In 2014 updates were made to the eTG and a new section on surgical prophylaxis of breast surgery was included in the guidelines. According to the current eTG, patients undergoing breast surgery should receive pre-operative cefazolin 2 grams intravenously (IV) within 60 minutes, ideally 15 to 30 minutes before surgical incision. For patients with immediate hypersensitivity to penicillin, vancomycin $15 \mathrm{mg} / \mathrm{kg} \mathrm{IV}$, started 30 to 120 minutes before surgical incision at a rate $10 \mathrm{mg} / \mathrm{min}$ is recommended [9]. Vancomycin should be added to cefazolin in suspected patients or patients infected with methicillin-resistant Staphylococcus aureus (MRSA) [9]. Post-operative antibiotics have not been indicated in these guidelines however the guidelines mention the potential benefits in obese patients, patients being treated with radiation therapy or in breast reconstruction patients [3, 8-14].

Prior to the update, prescribers would follow the protocol for 'Head, Neck and Thoracic' procedures [15]. The 2014 study conducted by Jaber et al., evaluated prescriber adherence to the eTG in breast surgeries performed at a single Western Australian teaching hospital prior to the 
77 guidelines update in 2014 [6]. Researchers reported low adherence to guidelines (13.3\%) and proposed an improvement would be seen after the update [6]. Since that time no study has been performed to evaluate the effectiveness of these updates nor an improvement in adherence.

\section{Aim of the study}

The aims of this study were to evaluate adherence to the eTG for antibiotic prophylaxis in breast surgery procedures at a Western Australian teaching hospital since the 2014 eTG update and to examine the impact of prophylactic antibiotics on SSI incidence and length of hospital stay.

\section{Ethical Approval}

The study was approved by the Sir Charles Gairdner Group Human Ethics and Research Committee (QI:14155) as a quality improvement activity to enhance the safety of medicines use. Approval of this piece of research included the option to publish the data with a waiver of individual patient consent. Ethical approval was also granted by the Human Research Ethics Committee at Curtin University, Perth, Western Australia (HRE2017-0189).

\section{Methods}

This retrospective cross-sectional study was conducted at Sir Charles Gairdner Hospital (SCGH) in Perth, Western Australia. A total of 973 patients who underwent a breast surgery procedure between February 2015 and March 2017 at SCGH were identified by the Pharmacy Department at SCGH using Medicare breast surgery codes by means of an electronic database. The search involved identifying patients with International Classification of Disease (ICD-10) codes. C50 was used as well as Australian Medicare Benefits Schedule principle procedures codes 31500 to 31566 which formed a list of patients not ordered by date, code or medical record number. Patients with missing important information such as patient treatment, detailed antibiotic use and type of surgery were excluded. In Australia, Medicare is a Commonwealth government universal health care system that provides citizens with access to many health services at minimal cost, including free treatment in public hospitals. The Medicare Benefits Schedule is a listing of the Medicare services subsidized by the Australian government. 
107 A sample of 250 patients was selected using an online randomiser [16]. This sample size was

108 selected so that the $95 \%$ confidence for the prevalence of guideline adherence would be no wider 109 than $+/-6 \%$. This degree of precision was considered adequate for this study. Only the first 110 operation for each patient was included for analysis. For patients whose files were unavailable, the 111 next patient sequentially from the random generator list was used.

112

113

114

115

116

117

118

119

120

121

122

123

124

125

126

127

128

129

130

131

132

133

134

135

136

De-identification of patient and prescriber details was performed during data collection to ensure confidentiality throughout the study. For this study details gathered from the medical records were entered into a password protected Microsoft Excel ${ }^{\circledR}$ Spreadsheet. Data extracted included patient demographics (age, gender, weight, height and allergies), the code of the surgeon who performed the procedure, the patients' ward, the procedure type, surgery time, any pre-operative or postoperative antibiotics given (drug name, dose, route of administration, time of administration and frequency of antibiotics given), adverse drug reactions for both pre- and post-operative antibiotics, signs of infection and the patient's length of stay in the hospital

In order to calculate adherence to the current version of the eTG, four characteristics of preoperative antibiotics recorded for each patient (antibiotic drug, dose, route of administration and timing of administration) were assessed against the current guidelines, which recommend the use of cefazolin 2 grams intravenously (IV) within 60 minutes, ideally 15 to 30 minutes before surgical incision [9]. To be classified as 'adherent' the prescriber must have satisfied all of these criteria of the eTG; if not, the treatment was rendered as 'non-adherent'. For all other determinations and statistical analysis in this study the Statistical Package for the Social Science (SPSS) version 23 software was utilised. The statistical significance of univariate associations between SSIs and adherence, procedure, prescriber, length of hospital stay (0-2 days vs 3 days or more), and the association between adherence and length of hospital stay were assessed using the Chi-square test or Fisher's Exact test, as appropriate. A Chi-square test was also used to compare levels of adherence to the eTG before and after the 2014 eTG update. A Logistic regression model was used to identify any multivariate associations with the dependent variables (guideline adherence, or development of a SSI). Results for the logistic regression are provided as the odds ratio, its $95 \%$ 
137 confidence interval $(95 \% \mathrm{CI})$, and p-value. A $p$-value $<0.05$ was taken to indicate a statistically

138 significant association in all tests.

139

140

\section{Results}

141 A total of 250 patients were analysed in this study. Most were females 98.8\% (247/250) (Table 1).

142 The $14.4 \%(36 / 250)$ patients who had a documented allergy to either penicillin or cefalosporins

143 were all female.

144

145

There were 25 different types of breast procedures recorded (Table 2). Many patients underwent

146

147

148

149

150

151

152

153

154

155

156

157

158

159

160

161

162

163

164

165

166

167 multiple procedures in the one operation with an average of 2.1 procedures/operation recorded per patient (median: 2 procedures/patient; range 1-4). A total of 521 procedures were performed during the 250 operations. The most common procedures performed were sentinel node biopsy $(n=120$; $23.0 \%)$ and wide local excision $(n=88 ; 16.9 \%)$. All male patients received a unilateral mastectomy with one male patient also receiving a sentinel node biopsy. There was no statistically significant association between any particular operation and development of a SSI.

There was missing information on specific adherence elements for 10.4\% (26/250) of patients, which related to timing of antibiotic administration. These patients were classified as noncompliant with respect to timing. Management of 123 patients $(49.2 \%)$ was found to be compliant with the eTG (Table 3). The 95\% CI for overall compliance was found to be: $43.0 \%-55.4 \%$. Adherence to specific factors of the eTG requirements were: correct drug 91.6\% (229/250), correct dose 53.6\% (134/250), correct route of administration 97.2\% (243/250) and correct timing of administration $86.4 \%(216 / 250)$ (Table 3). There was zero adherence to the eTG regarding patient allergies, although the allergies were not recorded as immediate hypersensitivity reactions to penicillin. Despite 37 patients reported as having an allergy to penicillin or cephalosporin, 67.6\% (25/37) received a cephalosporin (cefalozolin), 29.7\% (11/37) received clindamycin, and only one of these patients $(2.7 \%)$ did not receive an antibiotic.

Adherence to the eTG was found to be significantly improved since the study by Jaber et al. reported $13.3 \%$ compliance $(\mathrm{p}<0.001$, Chi-square test). In the current study, $5.2 \%(13 / 250)$ of patients developed a reported SSI after surgery. No statistically significant relationship was found 
168 between adherence to the eTG and SSIs (Odds ratio: $1.36 ; 95 \%$ CI: $0.43-4.30 ; p=0.597$ ). Of the 169 patients who had received appropriate pre-operative antibiotics treatment, $6.5 \%(8 / 123)$ developed 170 a reported SSI, while $4.9 \%(5 / 103)$ of the patients who did not receive appropriate treatment 171 developed an SSI.

172

173 On average patients stayed at the hospital for a mean ( \pm standard deviation) of $2.3 \pm 1.7$ days 174 (median: 2 days; range 1 - 16 days). With this length of stay divided into short (0-2 days) vs longer 175 (3 or more days), no significant association was found between level of adherence and length of 176 stay (OR: 2.0; 95\%CI: $0.5-7.6 ; p=0.3144)$ nor SSI occurrence $(p=0.596)$. A significant 177 relationship was found between using cefazolin and a decreased length of stay $(p=0.037)$, with $17887.8 \%$ (201/229) of those who were given cephazolin staying 1-2 days, compared to 71.4\% (15/21) 179 of those who were not taking cephazolin. There were 4.6\% (10/216) patients with a length of stay 180 less than three days who developed a SSI, whilst $8.8 \%(3 / 34)$ of those who stayed longer than two 181 days developed a SSI.

182

183

A total of seven surgeons were recorded in this study. There was no statistically significant 184 relationship found between prescribers and level of adherence $(p=0.631)$ with prescriber adherence 185 to the eTG ranging from 25 to $100 \%$. Similarly, between prescribers and developing an SSI 186 $(p=0.748)$ with individual SSIs rates for prescribers ranging from 0-14.3\%. No significance was found between prescribers and whether they gave pre-operative antibiotics with the rates of preoperative antibiotic given to patients for each prescriber ranging between $90.7 \%$ and $100 \%$. A statistically significant association was seen between prescriber and whether post-operative antibiotics were given $(p<0.05)$ with $11.2 \%$ (28/250) patients having received post-operative antibiotics despite not being specified on the eTG. A majority of patients who received postoperative antibiotics $(57.1 \% ; 16 / 28)$ were prescribed cefalexin, however other antibiotics including clindamycin $(14.3 \% ; 4 / 28)$, dicloxacillin $(10.7 \% ; 3 / 28)$, cefazolin $(3.6 \% ; 1 / 28)$, flucloxacillin $(3.6 \% ; 1 / 28)$, amoxicillin $(7.1 \% ; 2 / 28)$ amoxicillin with clavulanic acid $(3.6 \% ; 1 / 28)$ were also prescribed.

\section{Discussion}


198 This is the first study conducted since the introduction of the new eTG in 2014. The adherence 199 level of $49.2 \%$ (123 operations) was higher $(p<0.001)$ than the $13.3 \%$ (20 operations) reported by 200 Jaber et al. and zero adherence by Habak et al. who investigated adherence to the previous version 201 of the eTG [6,17]. This is an improved result compared to the previous study yet still not reflective 202 of strict adherence to the breast surgical antibiotic prophylaxis eTG guidelines. The main factor 203 that contributed to non-adherence, was antibiotic dosage which accounted for $46.4 \%$ of operations. 204 This was different to the study conducted by Jaber et al where inappropriate timing of the antibiotic 205 was the contributing factor for non-adherence [6]. To ensure adherence to guidelines, there are 206 measures that can be implemented as suggested by Nabor et al. [18]. These measures include 207 medical education presentations and laminated summaries of guidelines posted in surgical areas 208 such as operating rooms [18].

209

Antibiotic prophylaxis was used in $98.4 \%$ of patients who underwent a breast procedure which was higher than the $92.7 \%$ and 53\% reported by Jaber et al and Habak et al respectively [6,17]. Jaber et al. investigated the use of prophylactic antibiotic use in 150 breast operations in 150 patients whilst Habak et al. investigated prophylactic antibiotic use in 134 breast operations in 95 patients. Both studies were carried out in Western Australian hospitals [6, 17]. This shows an increased use of prophylactic antibiotics in the last two evaluations.

216

This study related to the oncological management of breast surgery as part of treatment. Despite the inclusion of one patient who had breast reduction and one patient who had implant insertion, these surgeries were related to an original life-saving procedure rather than a cosmetic procedure, as these are not performed at the study hospital. Antibiotics play an important role in these types of procedures as reported by Khan [19].

222

Of the 250 patients analysed in this study, 37 patients were recorded with an allergy to penicillin or cephalosporin, although there was no evidence that these were associated with immediate hypersensitivity reactions. It was found that none of the prescribers adhered to the eTG and had prescribed either cefazolin or clindamycin instead of vancomycin as stipulated in the eTG. Vancomycin or clindamycin are second-line choices for SSI prophylaxis in cases where cefalosporin antibiotics were contraindicated [20]. Possible reasons for reluctance by clinician to 
229 prescribe vancomycin include adverse effects such as red man syndrome which may limit its use

230 for some surgeons. Furthermore, vancomycin is contraindicated in patients with renal impairment $231[21]$.

232

233 No relationship was found between adherence to therapeutic guidelines and SSIs $(p=0.596)$. A recent study by Yang et al. also reported that there was no significance difference between SSIs and cefazolin administration in patients who underwent breast procedures such as a mastectomy, whereas development of SSIs with and without prophylactic pre-operative antibiotics was 7.2\% and $4.2 \%$ respectively [22]. In their paper they suggested that to decrease the development of postoperative SSIs other factors such as hypertension, diabetes, and advanced age should be taken into consideration [22].

While no significant association was found between the different operations and occurrence of a SSI, hematoma drainage has been clinically shown to impart an increased rate of SSIs for patients especially with a longer drain duration [23]. The study was able to collect data only on SSIs that were treated at the hospital. Other patients may have developed an SSI but were treated by a general practitioner or at another hospital.

There is a lack of consensus in current practice for the use of pre-operative antibiotic prophylaxis and this is largely attributed to the lack of trial evidence in preventing SSIs in general patients after breast surgery [24]. Also, the variation in guidelines and adherence can be attributed to changing patient risk factors. For instance, in a study by Eroglu et al. researchers reported that prescribers were more inclined to prescribe antibiotics prophylactically in patients with risk factors which included older age, diabetes mellitus, immunodeficiency and those who underwent pre-operative chemotherapy or radiotherapy prior to breast surgical procedures [23]. Other risk factors that may contribute to post-operative infections in breast surgery include length of surgery, type of surgery, smoking, steroid use, seroma, hematoma, surgical drain, second drain placed, prolonged close suction drainage and immediate breast reconstruction [25-33]. When risk factors are present in patients undergoing breast surgery, the administration of prophylactic antibiotics should be taken into account. This is supported by a recent study by Vieira et al. which reported that SSIs were 
259 significantly more common in the control group that did not receive antibiotic prophylaxis despite

260 having risk factors.[34]

261

262 For patients that are not at risk for SSIs after breast surgery, routine antibiotic prophylaxis is not 263 necessary [3, 25, 27, 32]. According to Xue et al. antibiotic prophylaxis is not an independent 264 protective factor in SSI development and systematic administration for breast surgery is not 265 necessary for general patients but may be considered if other risk factors were present [33].

In this present study no statistical significance was found between prescribers and adherence to 268 the eTG $(p=0.631)$. As mentioned previously there is a lack of clinical studies which support the use of antibiotic prophylaxis which may have influenced prescribers in the present study. The lack of consensus of antibiotic prophylaxis has also been reported by breast surgeons in the United Kingdom, following nationwide surveys. [24] The eTG does not indicate that antibiotic prophylaxis is discretionary [9].

273

Where patients were prescribed post-operative antibiotics, these did not adhere to the eTG in this study. Post-operative prophylaxis should not exceed 24 hours and should be considered on an individual patient basis. [9] Similar results have been reported by Jaber et al. and Habak et al. [6, 17]. According to other studies to prevent SSIs after breast and/or axillary surgery some surgeons prefer post-operative prophylaxis for patients with drains. In patients receiving a surgical drain, mastectomy, immediate reconstruction or receiving prior radiation therapy or chemotherapy, preand post-operative prophylactic antibiotics are used in these patients [25-27, 29, 32]. Phillips et al. [11], Manian, [8] Viola et al. [12], and Elbur et al.[13] each have emphasised the increased risk of antibiotic resistance and drug-related complications such as Clostridium difficile when postoperative antibiotic prophylaxis was used after breast surgeries, due to the lack of evidence.

It was also found that the mean length of stay was not influenced by the level of eTG adherence. However, the length of stay and SSIs were reduced when cefazolin was given. This somewhat supported the study conducted by Toor et al. who found that the administration of prophylactic antibiotics not only led to reduced SSIs but also led to a shortened hospital stay [35]. 
290 The strength of this study was a larger sample size $(n=250)$ compared to the previous study

291 conducted by Jaber et al. which used a sample size $(n=150)$. The results should be generalisable to

292 this hospital but may not reflect practice elsewhere.

293

294 Limitations of this study included the sample size $(n=250)$ which restricted the ability to identify

295 large numbers of SSIs. Patients might seek treatment for SSIs from their general practitioners or

296 other hospitals without returning to SCGH which may raise the incidence of SSIs recorded within

297 this study. A further limitation was that detailed examination of SSIs and the causative factors such

298 as pathogens, appropriate hand washing and other procedures, were beyond the scope of this study.

299 Also, missing information within 26 patient medical files regarding adherence, specifically the

300 timing of antibiotic administration meant these patients were excluded from the overall compliance

301 assessment.

302

303 Conclusions

304 There was a significant improvement in adherence to surgical antibiotic prophylaxis guidelines in

305 breast surgery from $13.3 \%$ to $49.2 \%$ compared to a similar study conducted by Jaber at al. [6].

306 Further improvement is necessary especially with respect to recording of antibiotic timing of

307 administration, adherence to guidelines for antibiotic dosage and also when allergy is reported to

308 the primary recommended antibiotic, that the recommended alternative antibiotic is selected. From

309 the available evidence, incidence of SSIs identified in the study was low and there was no

310 relationship with adherence to guidelines, indicating that the prophylaxis followed was reasonably

311 effective.

312

313 Acknowledgments

314 The authors acknowledge the assistance of the medical records department of the research hospital

315 for their support and assistance.

316

317

318 


\section{Reference list}

1. Cabaluna ND, Uy GB, Galicia RM, Cortez SC, Yray MD, Buckley BS. A randomized, double-blinded placebo-controlled clinical trial of the routine use of pre-operative antibiotic prophylaxis in modified radical mastectomy. World J Surg. 2013;37(1):59-66.

2. Craft RO, Damjanovic B, Colwell AS. Evidence-based protocol for infection control in immediate implant-based breast reconstruction. Ann Plast Surg. 2012; 69(4):446-450.

3. Gulluoglu BM, Guler SA, Ugurlu MU, Culha G. Efficacy of prophylactic antibiotic administration for breast cancer surgery in overweight or obese patients: a randomized controlled trial. Ann Surg. 2013; 257(1):37-43.

4. Ariyan S, Martin J, Lal A, Cheng D, Borah GL, Chung KC, Conly J, Havlik R, Lee W, McGrath MH, Pribaz J, Young VL. Antibiotic prophylaxis for preventing surgical-site infection in plastic surgery: an evidence-based consensus conference statement from the American Association of Plastic Surgeons. Plast Reconstr Surg. 2015; 135(6): 1723-1739.

5. Jones DJ, Bunn F, Bell-Syer SV. Prophylactic antibiotics to prevent surgical site infection after breast cancer surgery. Cochrane Database Syst Rev. 2014; 9(3).

6. Jaber S, Rogers C, Sunderland B, Parsons R, MacKenzie S, Seet J, Czarniak P. Appropriateness of surgical antibiotic prophylaxis for breast surgery procedures. Int J Clin Pharm. 2017; 39(2): 483-486.

7. Crawford CB, Clay JA, Seydel AS, Wernberg JA. Surgical site infections in breast surgery: the use of pre-operative antibiotics for elective, nonreconstructive procedures. Int J Breast Cancer. 2016.

8. Manian FA. The role of post-operative factors in surgical site infections: time to take notice. Clin Infect Dis. 2014; 59(9):1272-1276.

9. Antibiotic Expert Groups. Therapeutic guidelines: antibiotic. Version 14. Melbourne: Therapeutic Guidelines Limited; 2010.

10. Research Randomizer [Internet]. United States; c2013 [cited 2017 Sept 30]. Available from: https://www.randomizer.org/

11. Antibiotic Expert Groups. Therapeutic guidelines: antibiotic. Version 15. Melbourne: Therapeutic Guidelines Limited; 2014.

12. Huang N, Liu M, Yu P, Wu J. Antibiotic prophylaxis in prosthesis-based mammoplasty: a systematic review. Int J Surg. 2015; 15:31-37.

13. Phillips BT, Bishawi M, Dagum AB, Khan SU, Bui DT. A systematic review of antibiotic use and infection in breast reconstruction: what is the evidence? Plast Reconstr Surg. 2013; 131(1):1-13.

14. Viola GM, Raad II, Rolston KV. Breast tissue expander-related infections: perioperative antimicrobial regimens. Infect Control Hosp Epidemiol. 2014; 35(1):75-81.

15. Elbur AI, Yousif MA, Elsayed AS, Abdel-Rahman ME. An audit of prophylactic surgical antibiotic use in a sudanese teaching hospital. Int J Clin Pharm. 2013; 35(1): 149-153.

16. Townley WA, Baluch N, Bagher S, Maass SW, O'Neill A, Zhong T, Hofer SO. A single preoperative antibiotic dose is as effective as continued antibiotic prophylaxis in implant-based breast reconstruction: A matched cohort study. J Plast Reconstr Aesthet Surg. 2015; 68(5):673-678.

17. Habak JE, Varma S, Kiani M, Twaddle A, Emmerton LM. Prophylactic antibiotic use in breast cancer surgery patients. J Pharm Pract Res. 2013;43(2):101-4. 
18. Nabor MIP, Buckley BS, Lapitan MCM. Compliance with international guidelines on antibiotic prophylaxis for elective surgeries at a tertiary-level hospital in the Philippines. Healthcare Infect. 2015; 20(3-4): 145-151.

19. Khan UD. Breast Augmentation, Antibiotic Prophylaxis and Infection: Comparative Analysis of 1628 Primary Augmentation Mammoplasties to Assess the Role and Efficacy of Length of Antibiotic Prophylaxis. Aesth Plast Surg. 2010; 34(1):42-47.

20. Baghaki S, Soybir GR, Soran A. Guideline for antimicrobial prophylaxis in breast surgery. J Breast Health. 2014. 10(2): 79-82.

21. Bratzler DW, Dellinger EP, Olsen KM, Perl TM, Auwaerter PG, Bolon MK, Fish DN, Napolitano LM, Sawyer RG, Slain JP, Weinstein RA. Clinical practice guidelines for antimicrobial prophylaxis in surgery. Am J Health Syst Pharm. 2013; 70(3): 195-283.

22. Yang S, Liu G, Tang D, Cai D. Evaluation intravenous drip cefazolin prophylaxis of breast cancer surgery site infection. J Craniofac Surg. 2017;28(6):527-531.

23. Eroglu A, Karasoy D, Kurt H, Baskan S. National practice in antibiotic prophylaxis in breast cancer surgery. J Clin Med Res. 2014; 6(1):30-35.

24. Ng D, Trivedi PM, Sharma AK, Banerjee D. Current use of antibiotic prophylaxis in breast surgery: a nationwide survey. The Breast. 2007; 16(1):68-72.

25. Vilar-Compte CD, Jacquemin B, Robles-Vidal C, Volkow P. Surgical site infections in breast surgery: case-control study. World J Surg. 2004; 28(3):242-246.

26. Throckmorton AD, Boughey JC, Boostrom SY, Holifield AC, Stobbs MM, Hoskin T, Baddour LM, Degnim AC. Post-operative prophylactic antibiotics and surgical site infection rates in breast surgery patients. Ann Surg Oncol. 2009; 16(9): 2464-2469.

27. Tejirian T, DiFronzo LA, Haigh PI. Antibiotic prophylaxis for preventing wound infection after breast surgery: a systematic review and meta-analysis. J Am Coll Surg. 2006; 203(5):729-734.

28. Penel N, Yazdanpanah Y, Chauvet MP, Clisant S, Giard S, Neu JC, Lefebvre D, Fournier C, Bonneterre J. Prevention of surgical site infection after breast cancer surgery by targeted prophylaxis antibiotic in patients at high risk of surgical site infection. J Surg Oncol. 2007; 96(2):124-129.

29. De Blacam C, Ogunleye AA, Momoh AO, Colakoglu S, Tobias AM, Sharma R, Houlihan MJ, Lee BT. High body mass index and smoking predict morbidity in breast cancer surgery: a multivariate analysis of 26,988 patients from the national surgical quality improvement program database. Ann Surg. 2012; 255(3): 551-555.

30. Vilar-Compte D, Rosales S, Hernandez-Mello N, Maafs E, Volkow P. Surveillance, control, and prevention of surgical site infections in breast cancer surgery: a 5-year experience. Am J Infect Control. 2009; 37(8): 674-679.

31. Gao YX, Xu L, Ye JM, Wang DM, Zhao JX, Zhang LB, Duan XN, Liu YH. Analysis of risk factors of surgical site infections in breast cancer. Chin Med J (Engl). 2010; 123(5):559-562.

32. Olsen MA, Lefta M, Dietz JR, Brandt KE, Aft R, Matthews R, Mayfield J, Fraser VJ. Risk factors for surgical site infection after major breast operation. J Am Coll Surg. 2008; 207(3): 326-335.

33. Xue DQ, Qian C, Yang L, Wang XF. Risk factors for surgical site infections after breast surgery: a systematic review and meta-analysis. Eur J Surg Oncol. 2012; 38(5):375-381.

34. Vieira LF, De Melo Neto AF, Schio MR, De Oliveira JA, Almeida CL, Ferraz DM. Controversies in Reduction Mammoplasty: Being A "Clean" Operation, Does It Mandate Antibiotic Prophylaxis? Surg Infect. 2016; 17(5):596-600. 
410 35. Toor AA, Farooka MW, Ayyaz M, Sarwar H, Malik AA, Shabbir F. Pre-

411 operative antibiotic use reduces surgical site infection. J Pak Med Assoc. 2015; 65(7):733-

412

413 736. 


\section{Table $\mathbf{1}$ (on next page)}

Patient demographics 


\begin{tabular}{|l|lc|}
\hline \multicolumn{1}{|c|}{ Demographic } & \multicolumn{1}{c|}{ Gender } & Mean ( \pm standard deviation) \\
\hline \multirow{2}{*}{ Age (years) } & Female $(\mathrm{n}=247)$ & $56.8 \pm 13.7$ \\
& Male $(\mathrm{n}=3)$ & $46.7 \pm 22.9$ \\
Weight $(\mathrm{kg})$ & Female $(\mathrm{n}=246)$ & $76.1 \pm 20.4$ \\
& Male $(\mathrm{n}=3)$ & $92.7 \pm 10.8$ \\
Height $(\mathrm{cm})$ & Female $(\mathrm{n}=235)$ & $162.4 \pm 8.0$ \\
& Male $(\mathrm{n}=3)$ & $183 \pm 1.7$ \\
\hline
\end{tabular}

1

2

3

4 


\section{Table 2 (on next page)}

Types of procedures and association with surgical site infections (SSI). P-valueswere obtained from Fisher's Exact test, unless otherwise specified. 


\begin{tabular}{|lcll|}
\hline Type of Procedure & $\begin{array}{l}\text { Frequency of } \\
\text { procedure }\end{array}$ & $\begin{array}{l}\text { Frequency (\%) } \\
\text { of SSI }\end{array}$ & $\boldsymbol{p}$ \\
\hline Axillary node clearance & 52 & $1(1.9 \%)$ & 0.313 \\
Mastectomy (unilateral) & 84 & $4(4.8 \%)$ & 1.0 \\
Mastectomy (bilateral) & 12 & $2(16.7 \%)$ & 0.123 \\
Sentinel node biopsy & 120 & $5(4.2 \%)$ & $0.480 *$ \\
Wide local excision & 88 & $3(3.4 \%)$ & 0.552 \\
Hook wire local excision & 68 & $1(1.5 \%)$ & 0.196 \\
Excision & 27 & $2(7.4 \%)$ & 0.638 \\
Reconstruction/expanders & 6 & $0(0.0 \%)$ & 1.0 \\
Implant insertion & 1 & $0(0.0 \%)$ & 1.0 \\
Microdochectomy & 6 & $0(0.0 \%)$ & 1.0 \\
Duct excision & 5 & $0(0.0 \%)$ & 1.0 \\
Lumpectomy & 2 & $0(0.0 \%)$ & 1.0 \\
Seed removal & 1 & $0(0.0 \%)$ & 1.0 \\
Soft tissue biopsy & 3 & $1(33.3 \%)$ & 0.149 \\
Abscess drainage & 5 & $1(20.0 \%)$ & 0.236 \\
Abscess incision & 5 & $1(20.0 \%)$ & 0.236 \\
Breast reduction & 1 & $0(0.0 \%)$ & 1.0 \\
Hematoma drainage & 1 & $1(100 \%)$ & 0.052 \\
DIEP flap breast & 20 & $1(16.7 \%)$ & 0.277 \\
reconstruction & 6 & $0(0.0 \%)$ & 1.0 \\
Wound exploration & 2 & $0(0.0 \%)$ & 1.0 \\
Seed localization & 2 & $0(0.0 \%)$ & 0.608 \\
Excision biopsy & $0(0.0 \%)$ & 1.0 \\
Re-excision & $0(0.0 \%)$ & 1.0 \\
Lipofilling & $0(0.0 \%)$ & 1.0 \\
Liposuction & 1 & & \\
\hline
\end{tabular}

* p-value obtained from the Chi-square statistic 
Table 3(on next page)

Levels of adherence to specific elements of the Australian Antibiotic Therapeutic Guidelines in 250 operations (indicates element complied with; indicates element not complied with) 
1 Table 3. Levels of adherence to specific elements of the Australian Antibiotic Therapeutic

2 Guidelines in 250 operations ( $\square$ indicates element complied with; $\square$ indicates element

3 not complied with).

\begin{tabular}{|c|c|c|c|c|c|}
\hline \multicolumn{4}{|c|}{$\begin{array}{c}\text { Correct adherence to prescribing parameters when compared to } \\
\text { the Therapeutic Guidelines: Antibiotic Version } 15\end{array}$} & \multirow{2}{*}{\multicolumn{2}{|c|}{$\begin{array}{c}\begin{array}{c}\text { Number of } \\
\text { operations }\end{array} \\
(\mathbf{n}=\mathbf{2 5 0})(\%)\end{array}$}} \\
\hline \multirow[t]{11}{*}{ Antibiotic selection } & Route & Dose & Timing & & \\
\hline & & & & 123 & 49.2 \\
\hline & & & & 79 & 31.6 \\
\hline & & & & 11 & 4.4 \\
\hline & & & & 13 & 5.2 \\
\hline & & & & 2 & 0.8 \\
\hline & & & & 1 & 0.4 \\
\hline & & & & 12 & 4.8 \\
\hline & & & & 5 & 2.0 \\
\hline & & & & 1 & 0.4 \\
\hline & & & & 3 & 1.2 \\
\hline
\end{tabular}

4 